\title{
Optimization of Metal Fin Distributions in Latent Heat Storages
}

\author{
Bart Peremans ${ }^{1,2, *}$, Maarten Blommaert ${ }^{1,2,3, * *}$, and Martine Baelmans ${ }^{1,2}$ \\ 1 KU Leuven, Department of Mechanical Engineering, Leuven, 3001, Belgium, \\ *bart.peremans@kuleuven. be, \\ 2 EnergyVille, Genk, 3600, Belgium, \\ ${ }^{3}$ Flemish Institute for Technological Research (VITO), Mol, 2400, Belgium
}

\begin{abstract}
In this research, optimal fin distributions are presented for latent heat storages charged by a constant input power water flow. The limited input power results in non-uniform melting of the Phase Change Material (PCM). Therefore, new designs with non-uniform fin distributions provide the opportunity to outperform the ones with uniform distributions. In this paper, we show that different optimal fin distributions are found depending on the fin width, amount of fins and the input power. The gain in charging performance is discussed by comparing with latent heat storages without heat transfer enhancement.
\end{abstract}

Keywords: Latent heat storage, Phase Change Material, optimal fin distribution, adjoint optimization, optimal design

\section{Introduction}

Over the past years, latent heat storages have gained increased interest due to their superior storage capacity. High storage capacities are achieved as a result of the high latent heat of melting of Phase Change Materials (PCMs) [3]. However, the low thermal conductivity of PCMs limits the charging and discharging performance significantly, causing long charging and discharging times. Heat transfer enhancement techniques for PCM storages are extensively studied both experimentally and numerically [3]. Highly conductive fin structures are often employed as they extract the heat from the heat transfer fluid and easily conduct it into the PCM domain [1]. However, it has been experimentally observed that the charging power steeply drops in time [4]. As the PCM melts gradually its ability of absorbing heat from the heat transfer fluid decreases as a result of increased thermal diffusion lengths. Moreover, due to the limited input power and limited convection of the heat transfer fluid, the PCM does not melt uniformly.

** Maarten Blommaert is a postdoctoral research fellow of the Research Foundation Flanders (FWO) and VITO, the Flemish Institute for Technological Research. 
Therefore, uniform fin distributions can be outperformed by new designs with irregular fin distributions, as some spots could benefit from a higher fin density.

In this paper, optimal fin distributions are presented for heat transfer enhancement for latent heat storages charged by a constant heat input water flow. An adjoint-based optimization is presented for efficient gradient calculations. Design updates are performed by consecutive BFGS updates. The first section describes the model geometry, governing equations, and the solution method used. The second section presents the optimization problem and optimality conditions, resulting in the adjoint and design equations. The third section introduces constraints on the design variables and the analytic relation between design variables and mesh parameters. Subsequently, optimal fin distribution are presented for the simplified case of a PCM domain with one boundary at a constant wall temperature as well as for latent heat storages with heat supplied by a parallel plate water channel flow. The charging performance is compared to storages without fin structures. The last section provides a summary of the findings.

\section{Latent Heat Storage Modelling}

This section describes the geometry of the latent heat storage first. Secondly, the governing equations are shown which are solved to model the heat transfer in the storage.

\subsection{Model Geometry}

Latent heat storages are modelled in a two-dimensional domain, containing a heat transfer fluid channel and a PCM domain (see Fig. 1(a)). The heat transfer fluid channel with height $2 H$ is confined by metal walls with thickness $b_{w}$. The heat transfer fluid always flows from the left to the right. The PCM domain with dimensions $L_{x}$ and $L_{y}$ contains a single PCM embedded in a structure of rectangular metal fins. The fins have a fixed width $b_{f}$ and length $L_{y}$, and are perpendicularly mounted on the heat transfer fluid channel to enhance the heat transfer. The dashed line in Fig. 1(a) represents the symmetry plane. Therefore, only half of the storage is simulated.

Heat is supplied by a heat transfer fluid (water flow) with a fixed input power $\dot{Q}_{\text {in }}$ defined as

$$
\dot{Q}_{i n}=\dot{m} c_{w}\left(T_{i n}-T_{m}\right),
$$

with $\dot{m}$ the mass flow rate, $c_{w}$ the heat capacity, and $T_{i n}$ the input temperature of the water flow, and $T_{m}$ the melting temperature of the PCM. The latter serves as a reference temperature for potential sensible heat storage.

\subsection{Governing Equations}

To model the time-dependent heat storage problem, we consider a set of transport equations that describe the heat transport and storage in the PCM configuration, which we succinctly denote as $c(\mathbf{x}, \boldsymbol{\psi})=0$. These transport equations 


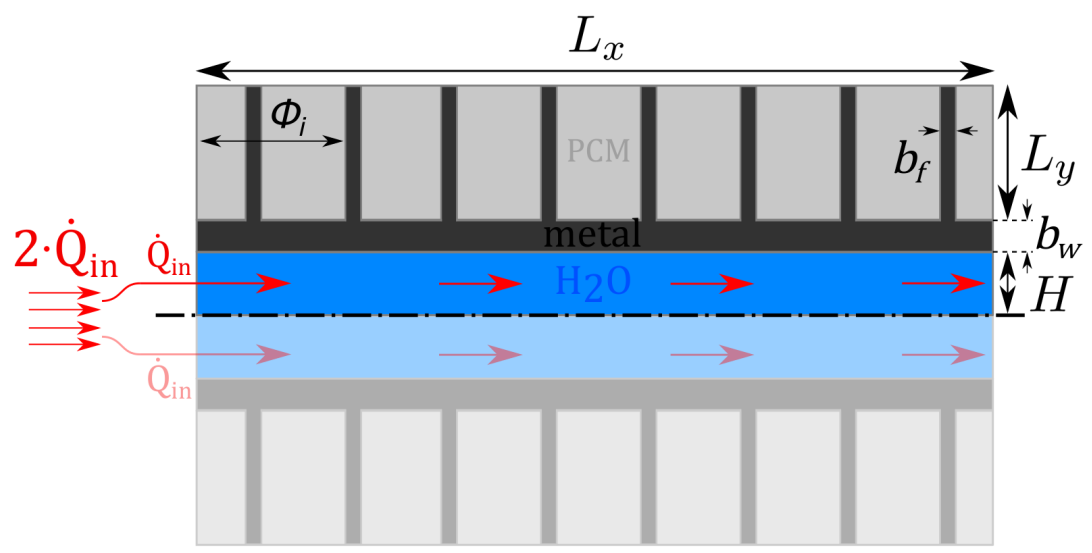

(a) Model geometry and design parameters.

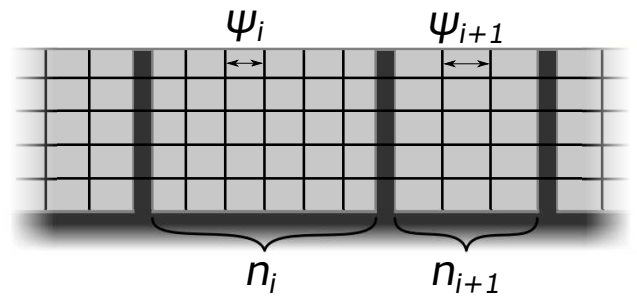

(b) Schematic representation of the grid and mesh parameters.

Fig. 1. 2D representation of a latent heat storage containing a heat transfer fluid channel, enclosed between metal plates, and rectangular PCM domain enhanced by metal fin structures. Only half of the storage is simulated with the dashed line as a plane of symmetry (a). The grid is constructed such that $n_{i}$ PCM cells have a width equal to the mesh parameter $\psi_{i}(\mathrm{~b})$.

include convective heat transfer in the water flow, and heat conduction and storage in PCM and metal structures. Here, $\mathbf{x}=\left[\begin{array}{l}\mathbf{h} \\ \mathbf{T}\end{array}\right]$ are the state variables for all time steps, which are found by solving the model equations $c(\mathbf{x}, \boldsymbol{\psi})=0$, after discretizing on a mesh with mesh parameters $\psi$. These mesh parameters correspond to the cell widths in the PCM domain (see Fig. 1(b)). The mesh parameters depend on the design variables $\phi$, i.e. the fin positions, through the following analytical relation:

$$
\forall j, j \in[1, N]: \phi_{j}=\sum_{i=1}^{j} n_{i} \psi_{i}+(j-1) b_{f},
$$

with $N$ the amount of fins, $b_{f}$ the fin widths, and $n_{i}$ the amount of PCM cells of cell width $\psi_{i}$ (see Fig. 1(b)). The mesh relation, which will be denoted by $\psi(\phi)$, 
is then found as

$$
\forall i, i \in[1, N]: \psi_{i}=\left\{\begin{array}{ll}
\frac{\phi_{i}}{n_{i}} & : i=1 \\
\frac{\phi_{i}-\phi_{i-1}-b_{f}}{n_{i}} & : i \neq 1
\end{array} .\right.
$$

Note that, although the overall mesh is not uniform, equal cell widths are used for PCM contained between the same fins.

Considering an implicit time-stepping scheme to resolve the time-dependence in the linear set of equations $c(\mathbf{x}, \boldsymbol{\psi})=0$, the subset of state variables $\mathbf{x}_{\mathbf{i}+\mathbf{1}}$ at time $t_{i+1}$ is governed by the algebraic set of equations

$$
A(\phi) \mathbf{x}_{i+1}=b\left(\mathbf{x}_{i}\right) .
$$

This set of equations is solved at discrete points in time with time steps $\Delta t$ up to a final charge time $t_{c h}$, i.e. the total time of heat supply. The adjoint (see Section 3.1) requires to rewrite Eq. (4) as

$$
A \mathbf{x}_{i+1}=b\left(\mathbf{x}_{i}\right)=b_{0, i}-B \mathbf{x}_{i}, \quad \text { or } \quad A \mathbf{x}_{i+1}+B \mathbf{x}_{i}=b_{0, i} .
$$

Assembling these set of equations for all time steps results in a system of equations

$$
\mathcal{A} \mathbf{x}-\mathbf{b}_{0}=0
$$

with

$$
\mathcal{A}=\left[\begin{array}{cccc}
A_{1} & & & \\
B_{2} & A_{2} & & \\
& \ddots & \ddots & \\
& & B_{t_{c h}} & A_{t_{c h}}
\end{array}\right], \quad \mathbf{x}=\left[\begin{array}{c}
\mathbf{x}_{1} \\
\mathbf{x}_{2} \\
\vdots \\
\mathbf{x}_{t_{c h}}
\end{array}\right], \quad \text { and } \quad \mathbf{b}_{0}=\left[\begin{array}{c}
b_{0,1} \\
b_{0,2} \\
\vdots \\
b_{0, t_{c h}}
\end{array}\right]
$$

PCM-metal structure Modelling the heat transfer in the PCM and metal requires solving the transient heat conduction equation, i.e.

$$
\rho \frac{\partial h}{\partial t}-\nabla \cdot k \nabla T=0
$$

with $\rho, c, h$ and $T$ the density, heat capacity, enthalpy and temperature of the PCM or metal. The phase change of the PCM is modelled using the enthalpy method:

$$
h=\left\{\begin{array}{ll}
c_{p, \mathrm{PCM}} T & : T<T_{m} \\
c_{p, \mathrm{PCM}} T+\Delta h & : T>T_{m}
\end{array} .\right.
$$

Water flow channel The heat transfer in the water flow is solved by a heightaveraged energy equation [2]

$$
\begin{array}{r}
\frac{\partial \rho_{w} c_{w}\langle T\rangle}{\partial t}+\langle\zeta \xi\rangle \frac{\partial}{\partial x}\left[\rho_{w} c_{w}\langle v\rangle\langle T\rangle\right]+(1-\langle\zeta \xi\rangle) \frac{\partial}{\partial x}\left[\rho_{w} c_{w}\langle v\rangle T_{i}\right] \\
-\frac{\partial}{\partial x}\left[k_{w} \frac{\partial\langle T\rangle}{\partial x}\right]-k_{w}\left(\langle T\rangle-T_{i}\right)\left\langle\frac{\partial^{2} \xi}{\partial y^{2}}\right\rangle=0,
\end{array}
$$


with the subscript $w$ denoting the water properties, subscript $i$ the water-metal interface, $\zeta(y)$ and $\xi(y)$ the imposed dimensionless velocity and temperature profile, $v$ the flow velocity, and $\langle$.$\rangle the averaging operator. To reduce the com-$ putational cost, the water flow is assumed to be fully hydrodynamically and thermally developed. Therefore, the flow equations are not included. The imposed velocity and temperature profile result in a constant factor $\langle\zeta \xi\rangle=1.2239$ and a quadratic dependent factor $\left\langle\frac{\partial^{2} \xi}{\partial y^{2}}\right\rangle=2.3072 H^{2}$.

\section{Adjoint-based Optimization}

This section first formulates the optimization problem and the coupling between design variables and mesh parameters. Second, the Lagrangian is constructed and the optimality conditions are derived, resulting in the adjoint and design equations.

\subsection{The Optimization Problem}

The optimization problem is mathematically formulated as

$$
\begin{aligned}
\min _{\mathbf{x}, \boldsymbol{\phi} \in\left[\phi_{\min }, \phi_{\max }\right]} & \mathcal{J}(\mathbf{x}, \psi(\phi)) \\
\text { s.t. } & c(\mathbf{x}, \psi(\phi))=0,
\end{aligned}
$$

with $\mathcal{J}$ the cost functional, $\mathbf{x}$ the state variables (i.e. temperature and enthalpy), $\phi$ the design variables (i.e. fin positions), and $\psi(\phi)$ the mesh relation as defined in section 2.2. $\phi_{\min }$ and $\phi_{\max }$ present lower and upper bounds for the design variables, further denoted as box constraints.

\subsection{The Lagrangian and Optimality Conditions}

The optimality conditions are derived by using Lagrange multipliers. The Lagrangian $\mathcal{L}$ without considering the box constraints is written as

$$
\mathcal{L}\left(\mathbf{x}, \mathbf{x}^{*}, \boldsymbol{\phi}\right)=\mathcal{J}(\mathbf{x}, \psi(\phi))+\mathbf{x}^{* T} c(\mathbf{x}, \psi(\phi)),
$$

with $\mathbf{x}^{*}$ the Lagrange multipliers. The first order necessary conditions for optimality (KKT-conditions) of the state-constrained optimization problem is found by equating the derivatives of the Lagrangian to zero, i.e.

$$
\left\{\begin{array}{l}
\nabla_{\mathbf{x}^{*}} \mathcal{L}=c(\mathbf{x}, \psi(\boldsymbol{\phi}))=0, \\
\nabla_{\mathbf{x}} \mathcal{L}=\nabla_{\mathbf{x}} \mathcal{J}(\mathbf{x}, \psi(\phi))+\frac{\partial c(\mathbf{x}, \psi(\phi))}{\partial \mathbf{x}}^{T} \mathbf{x}^{*}=0 \\
\nabla_{\boldsymbol{\phi}} \mathcal{L}=\nabla_{\boldsymbol{\phi}} \mathcal{J}(\mathbf{x}, \psi(\boldsymbol{\phi}))+\frac{\partial c(\mathbf{x}, \psi(\phi))}{\partial \boldsymbol{\phi}}^{T} \mathbf{x}^{*}=0
\end{array}\right.
$$

The first set of equations corresponds exactly to the state equations. The second set of equations are the adjoint equations and the corresponding Lagrange 
multipliers $\mathbf{x}^{*}$ are the adjoint variables. Replacing the governing equations $c$ by the time-stepping equations at a certain time (i.e. Eq. (6)) simplifies the adjoint equations to

$$
\nabla_{\mathbf{x}} \mathcal{J}(\mathbf{x}, \psi(\phi))+\mathcal{A}^{T} \mathbf{x}^{*}=0 .
$$

These equations can be solved efficiently for the adjoint variables $\mathbf{x}^{*}$ by marching back in time. The last set of equations are called the design equations. Applying the chain rule to the design equations and inserting Eq. (6) results in

$$
\nabla_{\boldsymbol{\phi}} \mathcal{L}=\frac{\partial \boldsymbol{\psi}}{\partial \boldsymbol{\phi}}^{T} \nabla_{\boldsymbol{\psi}} \mathcal{J}(\mathbf{x}, \psi(\boldsymbol{\phi}))+\frac{\partial \boldsymbol{\psi}^{T}}{\partial \boldsymbol{\phi}}\left(\frac{\partial \mathcal{A}}{\partial \boldsymbol{\psi}} \mathbf{x}-\frac{\partial \mathbf{b}_{0}}{\partial \boldsymbol{\psi}}\right)^{T} \mathbf{x}^{*}
$$

Since both the cost functional $\mathcal{J}$ and the state equations $c$ are explicitly expressed as a function of the mesh parameters $\boldsymbol{\psi}$, the derivatives $\nabla_{\boldsymbol{\psi}} \mathcal{J}(\mathbf{x}, \psi(\phi))$ and $\frac{\partial c(\mathbf{x}, \psi(\phi))}{\partial \psi}$ can be calculated analytically. To compute the derivatives $\frac{\partial \psi}{\partial \phi}$, the inverse relation between mesh parameters and design variables, i.e. Eq. (3), is needed. The derivatives are easily computed by analytically differentiating this functional.

\section{Fin Spacing Optimization}

In this section, the cost functional and the box constraints are discussed first. Secondly, the optimization method is discussed. Thirdly, the uniform fin distribution is verified as the optimal fin distribution for PCM domains heated by a constant wall temperature heat supply. Lastly, optimized fin distributions are presented for different cases, i.e. different fin widths and input powers.

\subsection{Cost functional and constraints}

Since we want to be able to charge the PCM as fast as possible for a given amount of PCM material, we reformulate the design problem as a mathematical minimization problem with an objective functional $\mathcal{J}$ defined as the negative of the mean charging power, i.e.

$$
\mathcal{J}(\mathbf{x}, \psi(\phi))=-\sum_{\forall i \in \Omega} \frac{(\rho V h)_{i}^{t=t_{c h}}}{t_{c h}} .
$$

with the index $i$ referring to a grid cell and $\Omega$ the PCM domain (including the metal fins). The amount of heat stored is evaluated at the final charge time. Recall that $\mathbf{x}=\left[\begin{array}{c}\mathbf{h} \\ \mathbf{T}\end{array}\right]$. Note that the cost functionals dependency on the mesh parameters is incorporated in the grid cell volumes $V_{i}$. The complete optimization problem is then formulated in its reduced form as

$$
\begin{array}{ll}
\min _{\phi} & \hat{\mathcal{J}}(\phi) \\
& h(\phi) \leq 0,
\end{array}
$$


with the reduced cost functional defined as $\hat{\mathcal{J}}(\boldsymbol{\phi})=\mathcal{J}(\mathbf{x}(\psi(\boldsymbol{\phi})), \psi(\boldsymbol{\phi}))$ and $h(\boldsymbol{\phi})$ including the box constraints. To compute the gradient in Eq. (15), the derivatives $\frac{\partial \psi}{\partial \phi}$ need to be derived analytically. Deriving Eq. (3) results in the following derivatives:

$$
\forall(i, j), i, j \in[1, N]: \frac{\partial \psi_{i}}{\partial \phi_{j}}= \begin{cases}\frac{1}{n_{i}} & : i=j \\ -\frac{1}{n_{i}} & : i=j+1 \\ 0 & : \text { else }\end{cases}
$$

Substituting Eq. (18) in the design equations (Eq. (15)) reduces the gradient calculation to

$$
\begin{array}{r}
\frac{\partial \mathcal{L}}{\partial \phi_{i}}=\left(\frac{\partial \psi_{i}}{\partial \phi_{i}} \frac{\partial \mathcal{J}}{\partial \psi_{i}}+\frac{\partial \psi_{i+1}}{\partial \phi_{i}} \frac{\partial \mathcal{J}}{\partial \psi_{i+1}}\right)+\left[\frac{\partial \psi_{i}}{\partial \phi_{i}}{\frac{\partial c^{T}}{\partial \psi_{i}}}^{T}+\frac{\partial \psi_{i+1}}{\partial \phi_{i}} \frac{\partial c}{\partial \psi_{i+1}}{ }^{T}\right] \mathbf{x}^{*} \\
=\left(\frac{1}{n_{i}} \frac{\partial \mathcal{J}}{\partial \psi_{i}}-\frac{1}{n_{i+1}} \frac{\partial \mathcal{J}}{\partial \psi_{i+1}}\right)+\left[\frac{1}{n_{i}} \frac{\partial c^{T}}{\partial \psi_{i}}-\frac{1}{n_{i+1}} \frac{\partial c}{\partial \psi_{i+1}}\right] \mathbf{x}^{*},
\end{array}
$$

for $i \in[1, N]$. Both the cost functional $\mathcal{J}$ as the model equations $c$ are explicit function of the variables $\boldsymbol{\psi}$. The derivatives $\frac{\partial \mathcal{J}}{\partial \psi}{ }_{i}$ and $\frac{\partial c}{\partial \psi}{ }_{i}$ are calculated based on Eq. (16) and Eq. (8)-(10).

The functional $h(\phi)$ describes practical constraints on the fin positions. The fin positions should lie inside the PCM domain. These constraints are analytically expressed using the box constraints with the lower and upper limits defined as

$$
\phi_{\min }=0 \quad \text { and } \quad \phi_{\max }=L_{x}-b_{f}
$$

\subsection{Optimization Algorithm}

In each optimization iteration, an improvement is sought for the fin positions. The steepest descent method was found to converge very slowly. Therefore, the BFGS method is applied for subsequent fin spacing updates [5]. The BFGS method computes an approximation of the Hessian of the optimization problem, i.e. $H \approx \mathcal{B}_{k}$, for every optimization iteration $k$. To ensure positive definiteness of the approximate Hessian $\mathcal{B}_{k}$, Powell's trick is applied. By including information about the Hessian, the BFGS method allows to converge faster to the optimal solution.

Note that the box constraints were not taken up into the adjoint derivation. To ensure these constraint are not violated, a step size $\mathbf{p}$ is calculated based on an SQP problem (Sequential Quadratic Programming) subject to constraints in which cost functional and constraints are linearised in each optimization iteration as a function of the step in design variables $p$, i.e.

$$
\begin{array}{cl}
\min _{\mathbf{p}} & \frac{1}{2} \mathbf{p}^{T} \mathcal{B} \mathbf{p}+\nabla \mathcal{J}\left(\boldsymbol{\phi}_{k}\right)^{T} \mathbf{p} \\
\text { s.t. } & h^{\prime}(\mathbf{p}) \leq 0
\end{array}
$$




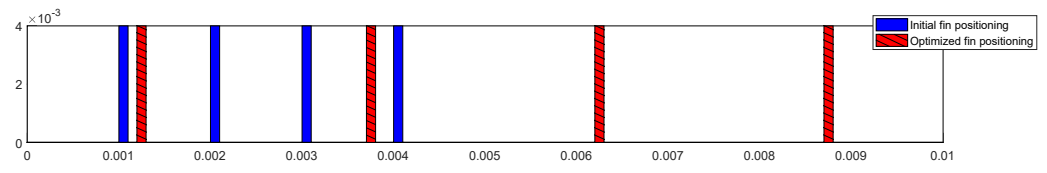

Fig. 2. Initial and optimized fin spacing of a PCM domain heated by a constant wall temperature boundary condition.

with $h^{\prime}(\mathbf{p})$ the practical constraints (Eq. (20)) translated to constraints for the step size $\mathbf{p}=\phi_{k+1}-\phi_{k}$ :

$$
h^{\prime}(\mathbf{p})=\left\{\begin{array}{l}
-\mathbf{p}-\phi_{k} \\
\mathbf{p}+\phi_{k}-L_{x}+b_{f}
\end{array} .\right.
$$

The fin spacing steps $\mathbf{p}_{k}$ for each iteration $k$ should satisfy the Armijo condition [5] to ensure a continuous decrease in cost functional, i.e.

$$
\mathcal{J}\left(\phi_{k}+\alpha_{k} \mathbf{p}_{k}\right) \leq \mathcal{J}\left(\phi_{k}\right)+\gamma \alpha_{k} \nabla \mathcal{J}\left(\phi_{k}\right)^{T} \mathbf{p}_{k},
$$

with $\alpha_{k}, \gamma \in[0,1]$ the relaxation factor for the step size and the relaxation of the gradient respectively. Therefore, a line search is used to determine a suitable relaxation factor $\alpha_{k}$ for each optimization iteration.

\subsection{Constant Wall Temperature Heat Source}

The first case to be considered is the simplified latent heat storage containing a PCM domain with a constant wall temperature boundary condition. It is stated in [1] that a uniform fin distribution shows the best charging performance. Therefore, the optimization method should lead to similar results.

A PCM domain with four fins is initialized with initial fin positions close to the left boundary (see Fig. 2). The optimal fin distribution is shown in Fig. 2 and exactly matches the uniform fin distribution. The statements in [1] are therefore verified by numerical optimization. However, a constant wall temperature is never achieved in practice. To simulate a more realistic case, a heat transfer fluid is included, in the next section.

\subsection{Heat Transfer Fluid as Heat Source}

In practical latent heat storages, heat is supplied by a heat transfer fluid with a finite total heat capacity, therefore limiting the available power. Because of the nature of this heat supply, the PCM will not melt uniformly. This already suggests that uniform fin distributions can be outperformed when including numerically optimized fin positions.

Previous research studied the effect of reshaping the PCM domain [2]. It was concluded that reducing the thickness of the PCM layer (i.e. $L_{y}$ ) is the 
Table 1. PCM properties.

\begin{tabular}{cc}
\hline & PCM \\
\hline density $\rho$ & $1000 \mathrm{~kg} / \mathrm{m}^{3}$ \\
heat capacity $c$ & $1000 \mathrm{~J} / \mathrm{kgK}$ \\
thermal conductivity $k$ & $0.2 \mathrm{~W} / \mathrm{mK}$ \\
latent heat of melting $\Delta h$ & $200 \mathrm{~kJ} / \mathrm{kg}$ \\
\hline
\end{tabular}

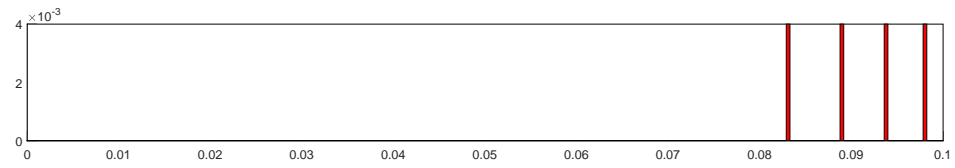

(a) 4 fins.

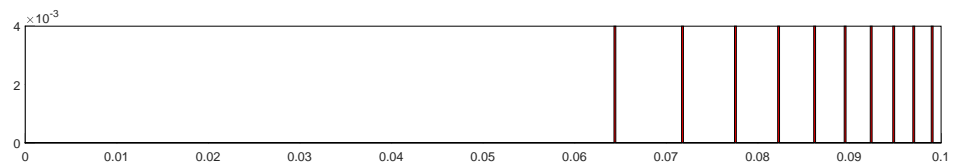

(b) 10 fins.

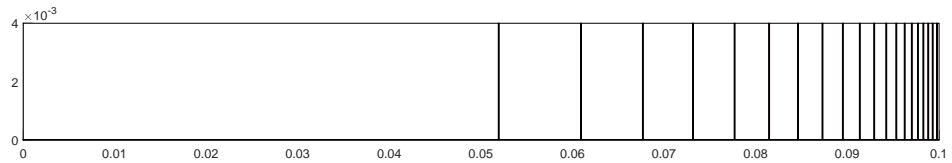

(c) 20 fins.

Fig. 3. Optimal fin spacing for different fin sizes, fixed metal volume fraction, and fixed input power $\left(\dot{Q}_{i n}=42 W\right)$.

most effective in enhancing the charging performance of the storage. Therefore, the optimization is performed for PCM domains with a high aspect ratio $\left(A R_{x y}=\frac{L_{x}}{L_{y}}=25\right.$, with $L_{x}=100 \mathrm{~mm}$ and $\left.L_{y}=4 \mathrm{~mm}\right)$. The shape of the water channel is fixed by the half-width $H=0.5 \mathrm{~mm}$ and metal wall thickness $b_{w}=0.2 \mathrm{~mm}$. The metal wall as well as the fins are made of aluminium. The aluminium fins are assumed to be rectangular and all equal in dimensions (length $L_{y}$, width $b_{f}$ ). The total fin volume is fixed to $2 \%$ of the PCM domain volume, i.e. $N \cdot b_{f}=0.02 L_{x}$, with $N$ the amount of fins. The properties of the PCM are listed in Table 1 and are representative for paraffins. The water supply temperature $T_{i n}$ is fixed to $10^{\circ} \mathrm{C}$ above the melting temperature $T_{m}$ of the PCM.

First, the optimization is performed for a fixed water mass flow rate, and thus input power (see Eq. (1)). However, the amount of fins and fin widths are altered. The charging time is fixed on $t_{c h}=2000 \mathrm{~s}$. The resulting optimal fin distributions for three cases, i.e. 4 fins, 10 fins and 20 fins, are shown in Fig. 3 and Fig. 4(a). For all cases, it is clear that the fins are pushed to the end of the PCM-domain (i.e. to the right in the figures). This is expected as the $\mathrm{PCM}$ on the left side of the domain is able to melt gradually, and therefore 


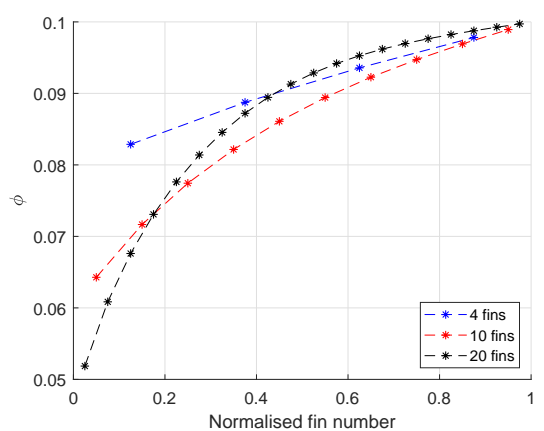

(a) Optimal fin distributions for different fin sizes.

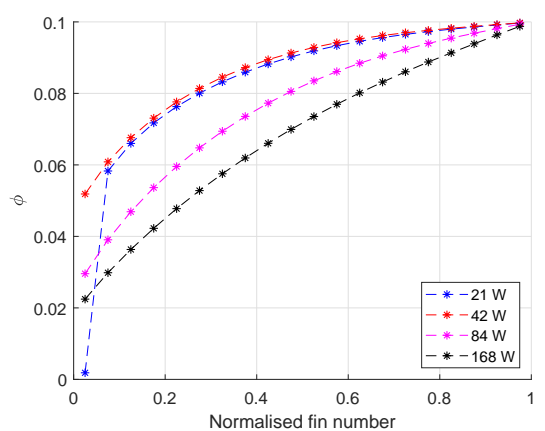

(b) Optimal fin distributions for different input powers.

Fig. 4. Optimal fin spacing optimization as a function of the normalised fin number $\left(=\frac{i-0.5}{N}\right.$ with $\left.i \in[1, N]\right)$ for different fin sizes (a) and input powers (b).

there is no need for heat transfer enhancement. In this part, an inclined phase change front is created, which evolves smoothly in the flow direction. Closer to the end of the channel, the fin density increases (i.e. the amount of fins per unit flow length) as to locally improve the charging characteristics. As a result, heat transfer takes place over a smaller flow length. In other words, the width of the phase change front (i.e. the size in the flow direction) reduces and the heat spreading in the water channel narrows. This prevents the output temperature of the water flow of increasing during charging for a longer period ${ }^{4}$. The smaller the fin widths and the higher the amount of fins, the more the fins are spread in the flow direction and the more they are concentrated towards the end of the channel. This is equivalent with the findings in [1], where smaller fins and more fins perform better with respect to charging. The fins increase the heat transfer surface, while encapsulating the PCM in small compartments, therefore greatly reducing the diffusion lengths.

Second, the effect of input power is investigated on the optimal fin positioning. Fig. 4(b) shows the fin distributions for three different input powers, achieved by altering the input mass flow rate of the water flow: $21 \mathrm{~W}, 42 \mathrm{~W}$, $84 \mathrm{~W}$ and $168 \mathrm{~W}$. The charging times are taken respectively $t_{c h}=4000 \mathrm{~s}, 2000 \mathrm{~s}$, $1000 \mathrm{~s}$ and $500 \mathrm{~s}$ to fix the same total heat input for all cases. The figures clearly show that the higher the heat input, the more the fins are spread over the domain. This is a result of wider phase change fronts due to higher input powers. Also, it is observed that slowly charging the latent heat storage is more effective as the charging ratio ${ }^{5}$ is higher (see Table 2). This is in accordance with

${ }^{4}$ Note that reduced charging performance results from increased output temperature of the water flow, as some of the available heat was not effectively stored and flows out of the channel.

5 The charging ratio is defined as the ratio of mean charging power to input power and defines the fraction of input heat that could effectively be stored. 
Table 2. Mean charging power, charging ratio, and enhancement factor for latent heat storages without fin structures and with optimized fin distributions for different fin amounts and input power. The enhancement factor is the ratio of the mean charging powers of the finned latent heat storage to the one of the storage without fins.

\begin{tabular}{ccccc}
\hline $\mathbf{N}$ & $\dot{Q}_{i n}$ & $\begin{array}{c}\text { Mean charging } \\
\text { power }\end{array}$ & Charging ratio & $\begin{array}{c}\text { Enhancement } \\
\text { factor }\end{array}$ \\
\hline 0 & $21 W$ & $18.95 W$ & 0.903 & - \\
0 & $42 W$ & $35.78 W$ & 0.852 & - \\
0 & $84 W$ & $62.89 W$ & 0.749 & - \\
0 & $168 W$ & $99.75 W$ & 0.594 & - \\
\hline \hline 4 & $42 W$ & $38.19 W$ & 0.909 & 1.067 \\
10 & $42 W$ & $40.22 W$ & 0.958 & 1.124 \\
20 & $21 W$ & $20.51 W$ & 0.977 & 1.082 \\
20 & $42 W$ & $40.96 W$ & 0.975 & 1.145 \\
20 & $84 W$ & $80.01 W$ & 0.953 & 1.272 \\
20 & $168 W$ & $144.05 W$ & 0.858 & 1.444 \\
\hline
\end{tabular}

the findings in [2]. Note that the lowest input power shows one fin completely shifted to the left of the PCM domain. It appears that 19 fins are sufficient for completely melting all of the PCM in the storage, i.e. the ones to the right. The other fin's position is optimized by means of maximizing the amount of sensible heat stored. Therefore, the fin is shifted to the left, close to the inlet of the channel where heat is being supplied.

The optimized fin distributions show that to achieve enhanced charging performance, fins should be placed more towards the end of the flow channel. Moreover, the fins should be spaced gradually closer to retrieve higher fin densities.

\section{Summary}

Fin distributions in latent heat storages were optimized to achieve improved charging properties. An adjoint-based optimization was presented for efficient gradient calculations. The BFGS method was applied for subsequent optimization updates. The presented fin distributions show a trend of shifting to the end of the water channel, as to conduct the heat to the most outer corner of the PCM domain. Inserting fins with smaller widths and therefore also a larger amount of fins, influences the optimal distribution and increases the charging performance. Also, different optimal fin distributions were found for varying input power. The optimal fin distributions all tend to gradually increase the fin density towards the end of the channel.

\section{References}

1. Peremans B., Blommaert M., Diriken J., Baelmans M.: Limits of latent heat storage charging. In: Proceedings of the 2nd Thermal and Fluid Engineering Conference, TFEC2017, Las Vegas (2017). 
2. Peremans B., Blommaert M., Diriken J., Baelmans M.: Towards energy efficient latent heat storage tanks with heat transfer fluid channels and finite inlet power. In: Proceedings of the 16th International Heat Transfer Conference, IHTC-16, Beijing (2018).

3. Zalba B., Marín J. M., Cabeza L. F., Mehling H.:Review on thermal energy storage with phase change: materials, heat transfer analysis and applications. In: Applied Thermal Engineering, vol. 23, pp. 251-283 (2003).

4. Gasia J., Diriken J., Bourke M., Van Bael J., Cabeza L. F.: Comparative study of the thermal performance of four different shell-and-tube heat exchangers used as latent heat thermal energy storage systems. In: Renewable Energy, vol. 144, pp. 934-944 (2017).

5. Nocedal J., Wright S. J.: Numerical optimization. Springer. 